\title{
SEQUENTIAL CLASSIFIER TRAINING FOR RICE MAPPING WITH MULTITEMPORAL REMOTE SENSING IMAGERY
}

\author{
Y. Guo ${ }^{\mathrm{a}}$, X. Jia ${ }^{\mathrm{a} *}$, D. Paull \\ ${ }^{a}$ School of Engineering and Information Technology, The University of New South Wales, Canberra, ACT 2600, Australia \\ - yiqing.guo@student.adfa.edu.au,x.jia@adfa.edu.au \\ ${ }^{\mathrm{b}}$ School of Physical, Environmental and Mathematical Sciences, The University of New South Wales, Canberra, ACT 2600, Australia \\ - d.paull@adfa.edu.au
}

KEY WORDS: Multitemporal Sentinel-2A Satellite Imagery, Classifier Training, Support Vector Machines (SVM), Rice Mapping, Spectral Index

\begin{abstract}
:
Most traditional methods for rice mapping with remote sensing data are effective when they are applied to the initial growing stage of rice, as the practice of flooding during this period makes the spectral characteristics of rice fields more distinguishable. In this study, we propose a sequential classifier training approach for rice mapping that can be used over the whole growing period of rice for monitoring various growth stages. Rice fields are firstly identified during the initial flooding period. The identified rice fields are used as training data to train a classifier that separates rice and non-rice pixels. The classifier is then used as a priori knowledge to assist the training of classifiers for later rice growing stages. This approach can be applied progressively to sequential image data, with only a small amount of training samples being required from each image. In order to demonstrate the effectiveness of the proposed approach, experiments were conducted at one of the major rice-growing areas in Australia. The proposed approach was applied to a set of multitemporal remote sensing images acquired by the Sentinel-2A satellite. Experimental results show that, compared with traditional spectral-indexbased algorithms, the proposed method is able to achieve more stable and consistent rice mapping accuracies and it reaches higher than $80 \%$ during the whole rice growing period.
\end{abstract}

\section{INTRODUCTION}

Continued mapping of rice fields with remote sensing data provides critical information for irrigation water budgeting and yield prediction (Kuenzer and Knauer, 2013, Mosleh et al., 2015). Periodically repeated observations of the same geographical area, known as multitemporal remote sensing, has enabled frequent monitoring of rice growth, and greatly facilitated studies on phenology and temporal transition of rice fields at a broad spatial scale.

Previous studies have shown that rice fields can be identified by detecting their unique optical features during the initial flooding period (Xiao et al., 2005). These spectral-index-based methods have been developed and applied for mapping rice distributions (Li et al., 2016, Zhang et al., 2015, Qin et al., 2015, Jin et al., 2016, Zhou et al., 2016, Dong et al., 2016). The Land Surface Water Index (LSWI), which is sensitive to leaf water and soil moisture, has mostly been utilized to enhance the spectral features of rice during the initial flooding period. Then discriminant rules have been set for the relationships between LSWI and biomass-related indices, such as Normalized Difference Vegetation Index (NDVI) and Enhanced Vegetation Index (EVI), to distinguish rice fields from other crops. However, these methods are only applicable to the initial flooding period of rice. Rice mapping during later rice growing stages is relatively harder, as the decreased water level and increased plant height make the spectral characteristics of rice less distinguishable from those of other crops.

In this study, we propose a sequential classifier training approach for rice mapping that can be applied to the whole growing period

${ }^{*}$ Corresponding author of rice. Rice fields are firstly identified during the initial flooding period. The identified rice fields are used as training data to train a classifier that separates rice and non-rice pixels. The classifier is then used as a priori knowledge to assist the training of classifiers for later rice growing stages. This approach can be applied progressively to sequential image data, with only a small amount of training samples being required from each image. In order to demonstrate its effectiveness, the proposed approach was applied to a set of multitemporal remote sensing images over one of the major rice-growing areas in Australia.

\section{METHOD}

\subsection{Proposed Procedure}

The proposed procedure for sequential classifier training is shown in Fig. 1. According to the sequence of sensing date, images during the flooding period are indexed $t-1, t-2, \cdots, t-N$, where $N$ is the total number of images during this period, and images during later rice growing stages are indexed $t, t+1, \cdots$. Firstly, rice fields are identified with spectral indices during the initial flooding period. The identified rice fields are used as training data to train a classifier that separates rice and non-rice pixels. The classifier is then used as an assistant classifier to assist the classifier training of Image $t$. A domain adaptation algorithm named Temporal-Adaptive Support Vector Machine (TASVM) (Guo et al., 2017) is adopted to train a classifier for Image $t$ by synthesizing the knowledge provided by the assistant classifier and the training samples. Then, the classifier for Image $t$ can be used as the updated assistant classifier to assist the training of the next image (Image $t+1$ ). By repeating this procedure, the proposed approach can be applied progressively to sequential image data. 


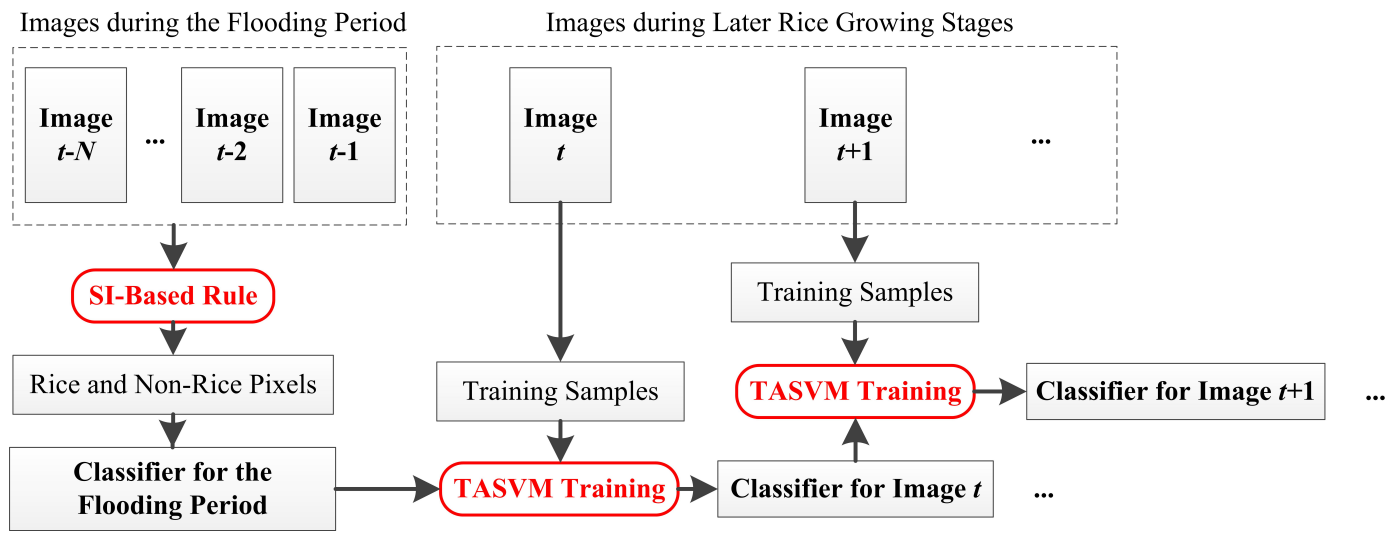

Figure 1. The proposed sequential classifier training procedure for rice mapping. SI: Spectral Index; TASVM: Temporal-Adaptive Support Vector Machine.

Detailed descriptions of the proposed approach are provided in the following two subsections.

\subsection{Rice Field Identification}

A well-established method for rice field mapping using optical remote sensing data is the spectral-index-based algorithms (Xiao et al., 2005, Xiao et al., 2006). These algorithms utilize the unique spectral features of rice fields during their initial flooding period, when rice plants are partially or entirely submerged under water. During this period, water surface contributes a significant amount of reflected solar radiance to the sensor. This leads to an increase in Land Surface Water Index (LSWI) and decreases in Normalized Difference Vegetation Index (NDVI) and Enhanced Vegetation Index (EVI). These indices are defined as:

$$
\begin{gathered}
\mathrm{LSWI}=\frac{\rho_{\text {nir }}-\rho_{\text {swir }}}{\rho_{\text {nir }}+\rho_{\text {swir }}}, \\
\mathrm{NDVI}=\frac{\rho_{\text {nir }}-\rho_{\text {red }}}{\rho_{\text {nir }}+\rho_{\text {red }}}, \\
\mathrm{EVI}=2.5 \times \frac{\rho_{\text {nir }}-\rho_{\text {red }}}{\rho_{\text {nir }}+6 \times \rho_{\text {red }}-7.5 \times \rho_{\text {blue }}+1},
\end{gathered}
$$

where $\rho_{\text {blue }}, \rho_{\text {red }}, \rho_{\text {nir }}$, and $\rho_{\text {swir }}$ are reflectance values in the blue, red, near-infrared, and short-wave-infrared bands, respectively. In this study, Band $2(490 \mathrm{~nm})$, Band $4(665 \mathrm{~nm})$, Band $8(842 \mathrm{~nm})$, and Band $11(1610 \mathrm{~nm})$ of the Sentinel-2A data are associated with the blue, red, near-infrared, and short-waveinfrared bands, respectively.

The rationale behind the spectral-index-based algorithms for rice field identification is further explained in Fig. 2. Due to the spectral characteristics of crop canopy, non-flooded crops such as maize (Fig. 2a) usually have a low reflectance value in the red band, a high reflectance value in the near-infrared band, and a medium reflectance value in the short-wave-infrared band. For the flooded rice field (Fig. 2b), its spectral characteristics are heavily affected by the presence of water. It can be observed that reflectance in the red and near-infrared bands decease, mainly due to the submersion of crop leaves. The reflectance in the shortwave-infrared band deceases as well because of the water absorption effect in this band.

Based on this rationale, the following spectral-index-based rule can be applied to identify rice fields (Dong et al., 2015):

$$
\mathrm{LSWI} \geq \min \{\mathrm{NDVI}, \mathrm{EVI}\}
$$

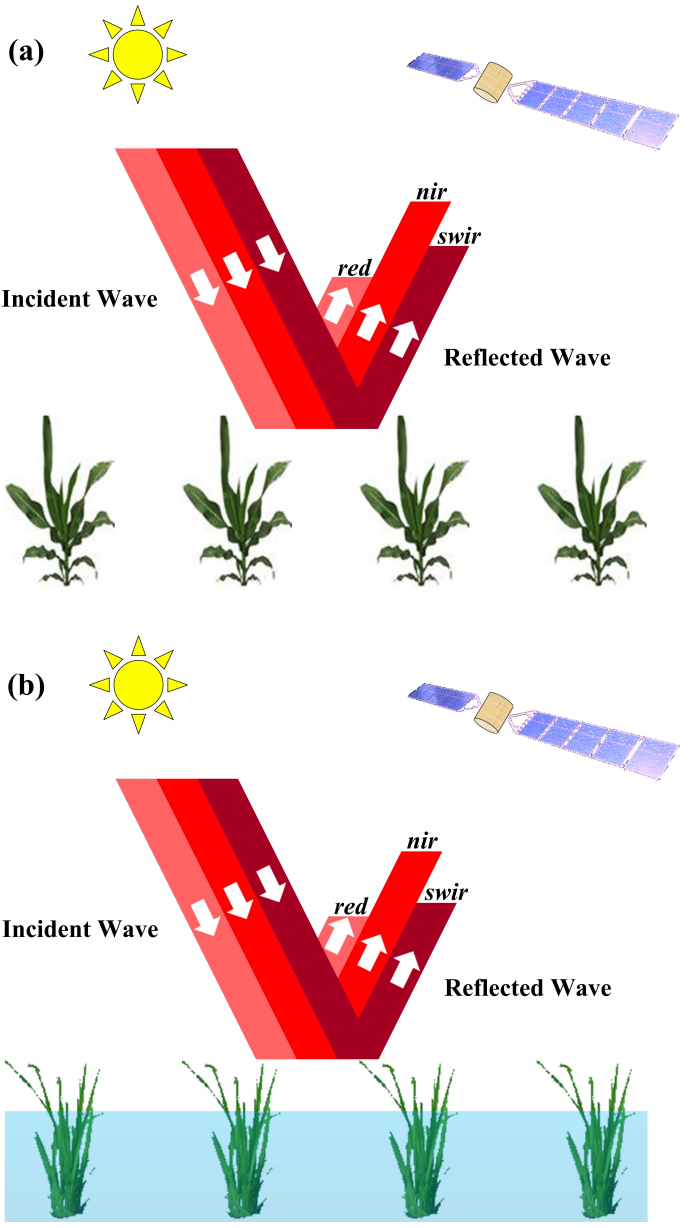

Figure 2. Reflectance characteristics in the red, near-infrared (nir), and short-wave-infrared (swir) bands for (a) non-flooded cropland (maize field) and (b) flooded rice field.

The water-sensitive index LSWI is compared with the biomasssensitive indices NDVI and EVI to distinguish flooded rice fields from other crops.

\subsection{Sequential Classifier Training}

The rice and non-rice fields identified with the spectral-indexbased rule in Eq. (4) are used to train a linear classifier $f_{\mathrm{s}}(\mathbf{x})$ 
that has the following form:

$$
f_{\mathrm{s}}(\mathbf{x})=\mathbf{w}_{\mathrm{s}}^{\mathrm{T}} \mathbf{x}+b_{\mathrm{s}},
$$

where $\mathbf{x}=\left(\begin{array}{llll}x_{1} & x_{2} & \cdots & x_{m}\end{array}\right)^{\mathrm{T}}$ is the feature vector with $m$ spectral features; $\mathbf{w}_{\mathrm{s}}=\left(w_{\mathrm{s}, 1} w_{\mathrm{s}, 2} \cdots w_{\mathrm{s}, m}\right)^{\mathrm{T}}$ is the weight vector and $b_{\mathrm{s}}$ is the bias factor for $f_{\mathrm{s}}(\mathbf{x})$.

We use $f_{\mathrm{s}}(\mathbf{x})$ to assist the training of a classifier for Image $t$ that has the following form:

$$
f_{\mathrm{t}}(\mathbf{x})=\mathbf{w}_{\mathrm{t}}^{\mathrm{T}} \mathbf{x}+b_{\mathrm{t}}
$$

where $\mathbf{w}_{\mathrm{t}}=\left(w_{\mathrm{t}, 1} w_{\mathrm{t}, 2} \cdots w_{\mathrm{t}, m}\right)^{\mathrm{T}}$ is the weight vector and $b_{\mathrm{t}}$ is the bias factor for $f_{\mathrm{t}}(\mathbf{x})$.

In addition to the assistant classifier $f_{\mathrm{s}}(\mathbf{x})$, the training samples of Image $t,\left\{\mathbf{x}_{i}, y_{i}\right\}_{i=1}^{n}$, are also utilized in the training of $f_{\mathrm{t}}(\mathbf{x})$, where $\mathbf{x}_{i}$ is the feature vector of the $i$ th training sample and $y_{i}$ is its corresponding label. The TASVM (Guo et al., 2017) is used to train $f_{\mathrm{t}}(\mathbf{x})$ by solving the following constrained quadratic programming problem:

$$
\begin{aligned}
\min _{w_{\mathrm{t}, j}, \xi_{i}, \mu_{j}} & \frac{1}{2} \sum_{j=1}^{m} w_{\mathrm{t}, j}{ }^{2}+C \sum_{i=1}^{n} \xi_{i}+F \sum_{j=1}^{m} \mu_{j} \\
\text { s.t. } \quad & y_{i}\left[\sum_{j=1}^{m} w_{\mathrm{t}, j} x_{i, j}+b_{\mathrm{t}}\right] \geq 1-\xi_{i} \text { and } \xi_{i} \geq 0, \forall i, \\
& -\mu_{j} \leq w_{\mathrm{t}, j}-w_{\mathrm{s}, j} \leq \mu_{j} \text { and } \mu_{j} \geq 0, \forall j,
\end{aligned}
$$

where $m$ and $n$ are the numbers of spectral features and training samples, respectively; $\mu=\left\{\mu_{j}\right\}_{j=1}^{m}$ and $\xi=\left\{\xi_{i}\right\}_{i=1}^{n}$ are two sets of non-negative slack variables.

The knowledge provided by $f_{\mathrm{s}}(\mathbf{x})$ and $\left\{\mathbf{x}_{i}, y_{i}\right\}_{i=1}^{n}$ are synthesized in Eq. (7) with two constraints. The first constraint restricts $f_{\mathrm{t}}(\mathbf{x})$ from departing too far away from the assistant classifier $f_{\mathrm{s}}(\mathbf{x})$. The second constraint measures the classification error of $f_{\mathrm{t}}(\mathbf{x})$ on the training samples $\left\{\mathbf{x}_{i}, y_{i}\right\}_{i=1}^{n}$. With the help of the assistant classifier $f_{\mathrm{s}}(\mathbf{x})$, only a small number of training samples are required for training $f_{\mathrm{t}}(\mathbf{x})$.

There are three terms in the objective function of Eq. (7). The first term accounts for the margin space of $f_{t}(\mathbf{x})$. The second and third terms aim to minimize the degree of violation of the constraints. The regularisation parameters $C$ and $F$ control the weights of the second and third terms relative to the first term.

The problem in Eq. (7) can be solved with standard quadratic programming algorithms (Nocedal and Wright, 2006). After determining $f_{\mathrm{t}}(\mathbf{x})$, it can be used as the updated assistant classifier to assist the training an classifier for the next image (Image $t+1$ ).

\section{EXPERIMENT AND RESULTS}

\subsection{Study Area and Data Sets}

3.1.1 Description of the study area The study focused on one of the major rice-growing areas in the Riverina region, the Coleambally Irrigation Area, which is located in the southwestern part of New South Wales, Australia, as shown in Fig. 3. The area has a semiarid climate, with distinct seasonal changes in temperature. The hottest month is January with an average $\mathrm{min} / \mathrm{max}$ temperature of $18.8 / 34.1{ }^{\circ} \mathrm{C}$, while the coldest month is July with an average $\min / \max$ temperature of $4.9 / 14.4{ }^{\circ} \mathrm{C}$. The annual rainfall is about $400 \mathrm{~mm}$, distributed evenly throughout the year. During summer, rice is the major crop in this area. A typical rice calendar is shown in Fig. 4. The whole growing period of rice lasts for 56 months, which can be divided into three major growth phases: the vegetative phase, the reproductive phrase, and the ripening phrase. The sowing date for rice seeds is during mid-late October, followed by several flush floodings and quick drains. Harvest of rice is during March to May of the second year. Different rice varieties are planted in the area, including Reiziq, Sherpa, Koshihikari, Opus, Illabong, Langi, Doongara, Kyeema, and Topaz. The other summer crops in this area are mainly maize, sorghum, and soybean, while winter wheat and citrus trees are the main winter crop and the main permanent crop, respectively. Due to the semi-arid climate and the high water consumption of the rice industry, water supplement in this area largely depends on irrigation from the Murrumbidgee River. Therefore, timely and accurate mapping of the spatial distribution of crop plantations is critical for the local Irrigation Company to make appropriate decisions on water budgeting and allocation.

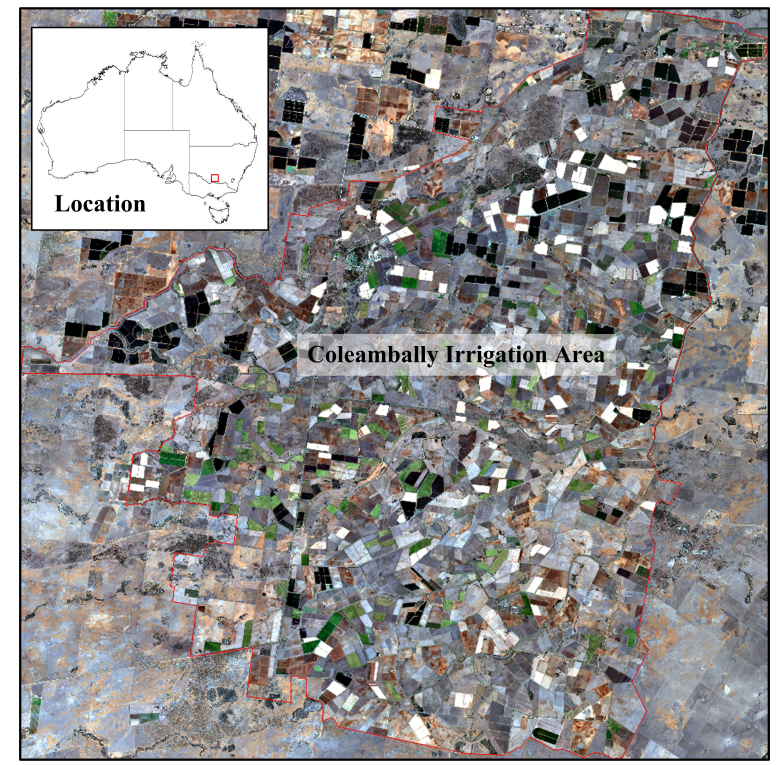

Figure 3. Location and color composite map (R: Band 4; G: Band 3; B: Band 2) of the study area.

3.1.2 Remote sensing images Optical remote sensing images provided by the recently launched Sentinel-2A satellite were used in this study. The images were downloaded from the Copernicus Open Access Hub (https://scihub.copernicus.eu/dhus/\#/home). A total of eight cloud-free images were available during the 2015-2016 rice growing season, as show in Fig. 4. Among them, the first four images covered the flooding period, while the later four images were captured during later rice growing stages. Each image consisted of 13 spectral bands covering the visible, near-infrared, and shortwave-infrared spectral regions. The images were provided in top of atmosphere (TOA) reflectance values with radiometric and geometric corrections applied (Level1C data). The TOA reflectances were then converted into atmospherically corrected bottom of atmosphere (BOA) reflectances (Level-2A data) with the Sentinel-2 Toolbox on the Sentinel Application Platform (SNAP). Terrain and cirrus corrections were also applied during the conversion process. After the conversion, the Cirrus band (Band 10) was omitted because no ground surface information was contained in this band. The bands with 20 $\mathrm{m}$ (Bands 5, 6, 7, 8a, 11, and 12) or $60 \mathrm{~m}$ (Bands 1 and 9) spa- 


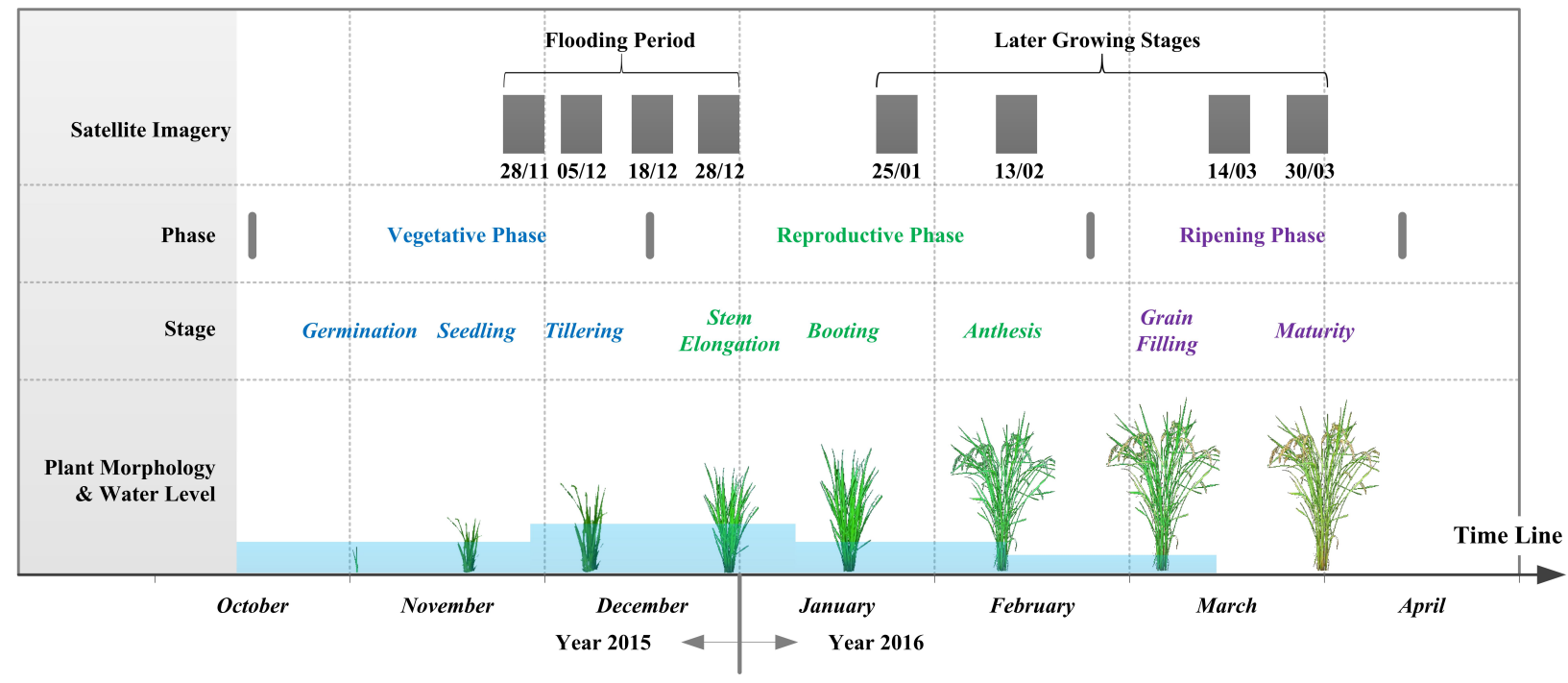

Figure 4. Rice calendar of the study area.
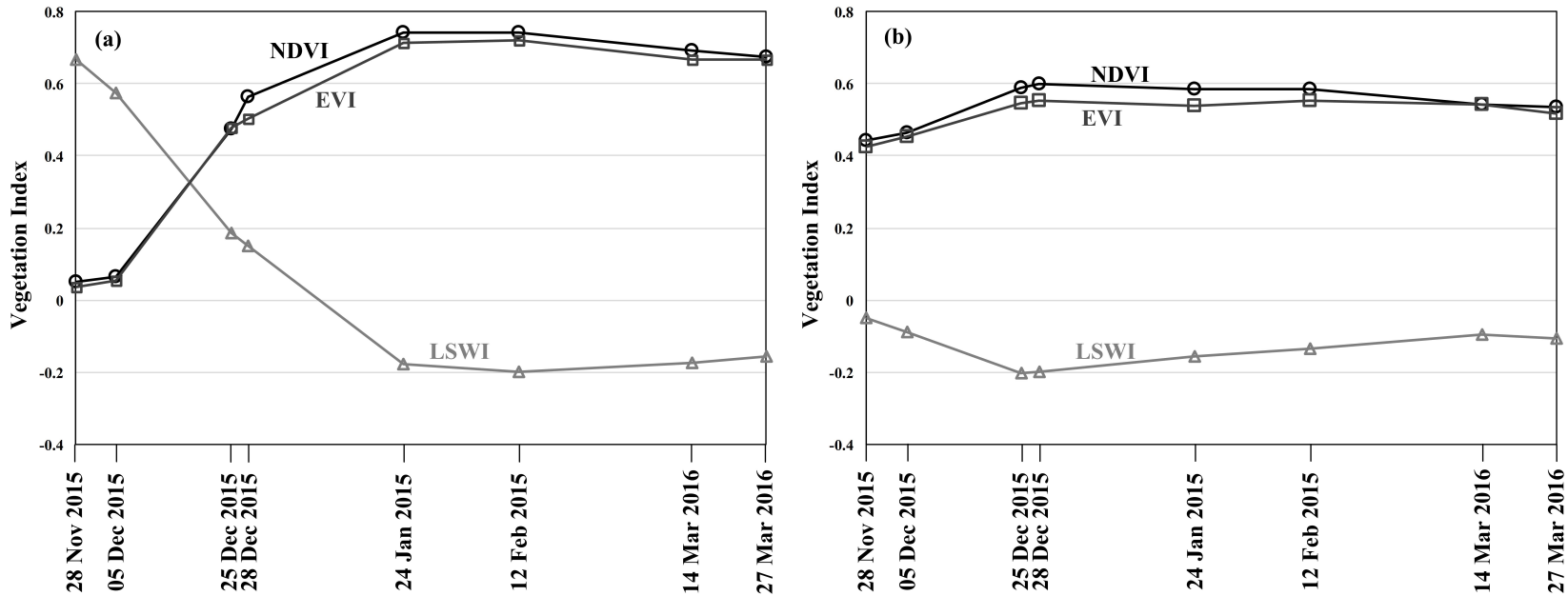

Figure 5. Temporal variations of Land Surface Water Index (LSWI), Normalized Difference Vegetation Index (NDVI), and Enhanced Vegetation Index (EVI) for (a) rice and (b) non-rice (citrus) crops.

tial resolutions were resampled into $10 \mathrm{~m}$ resolutions in order to make them consistent with the other bands that had $10 \mathrm{~m}$ resolutions (Bands 2, 3, 4, and 8).

3.1.3 Ground reference data The rice distribution map of the study area was provided by the local Coleambally Irrigation Company. The rice fields were identified via visual interpretation of a high spatial resolution image and several field visits during December 2015. In this study, these data were used as ground reference data to assess the accuracy of the proposed method.

\subsection{Results and Discussions}

3.2.1 Temporal variations of rice and non-rice crops The temporal variations of LSWI, NDVI, and EVI for a rice field and a non-rice field (citrus trees) are shown in Figs. 5a and 5b, respectively. It was found that, during the initial flooding period (the first two images), the LSWI of rice was higher than NDVI and EVI, which was different from that of the non-rice field. By using traditional spectral-index-based rules, rice fields could be distinguished from non-rice fields during this period. However, during the later growing stages, the LSWI of rice became lower than NDVI and EVI, which is similar to that of the non-rice field.
This made rice fields less distinguishable from non-rice fields. Therefore, traditional spectral-index-based rules are suitable for the initial flooding periods of rice.

3.2.2 Rice mapping accuracy Rice mapping accuracies obtained by the proposed method were compared with those by directly applying the traditional spectral-index-based rule to each image. The results are shown in Fig. 6. It was found that during the initial flooding period, the traditional method achieved reasonable rice mapping accuracies. However, decreased accuracies were observed for the traditional method during later rice growing periods. Compared with the traditional method, the proposed method achieved more stable and consistent rice mapping accuracies that were greater than $80 \%$ during the whole rice growing period. The results show the advantage of the proposed method in rice mapping during late rice growing period.

\section{CONCLUSIONS}

A sequential classifier training approach has been proposed for rice mapping that can be applied to the whole growing period of rice. The proposed method makes use of the effective identifi- 


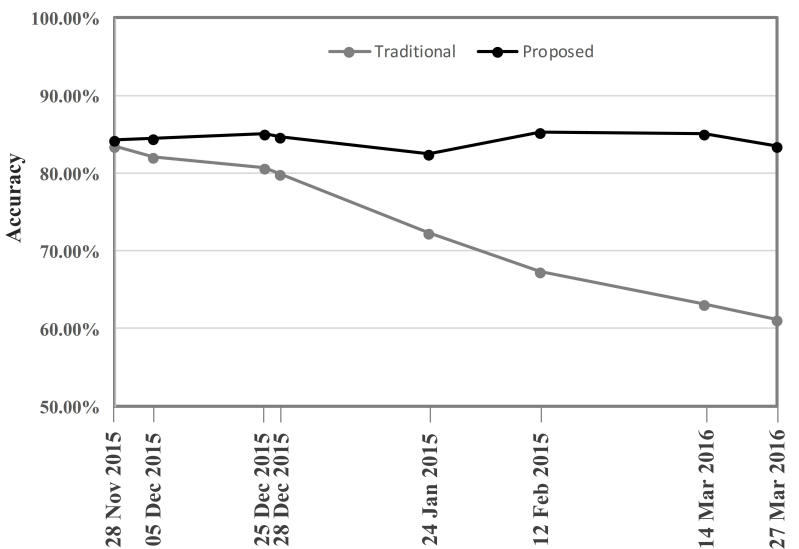

Figure 6 . Rice mapping accuracies obtained by the traditional and proposed methods.

cation results at the initial flooding period using spectral-indexbased algorithms. Training data for later images can then be collected with less intensive workload. The sequential training can be conducted with limited new training samples to reflect the current variations. Experimental results showed that, compared with the traditional spectral-index-based algorithms, the proposed method was able to achieve more stable and consistent rice mapping accuracies that were than $80 \%$ during the whole rice growing period.

As demonstrated in the experiment, the proposed rice mapping approach can be applied progressively to sequential image data over the whole growing period of rice. For classifier training of each incoming image, the classifier of the previous image is used as an assistant classifier, which reduces the required number of training samples. The assistant classifier can be more reliable if two images are sensed within a shorter temporal interval. When compared with other approaches that require the availability of raw data (pixels values or/and their corresponding labels) from the previous image, this approach is more flexible and computationally efficient as only the parameters of the assistant classifier are required and used for computation. A limitation of the current approach is that the land cover classes need to remain unchanged over time within the imaged area. So future studies need to be focused on solving this problem.

\section{ACKNOWLEDGEMENT}

The authors would like to thank Mr. Graham Parton and Mr. Bernard Star at the Coleambally Irrigation Co-operative Ltd for providing ground reference data of the Coleambally Irrigation Area.

\section{REFERENCES}

Dong, J., Xiao, X., Kou, W., Qin, Y., Zhang, G., Li, L., Jin, C., Zhou, Y., Wang, J., Biradar, C. et al., 2015. Tracking the dynamics of paddy rice planting area in 1986-2010 through time series Landsat images and phenology-based algorithms. Remote Sensing of Environment 160, pp. 99-113.

Dong, J., Xiao, X., Menarguez, M. A., Zhang, G., Qin, Y., Thau, D., Biradar, C. and Moore, B., 2016. Mapping paddy rice planting area in northeastern Asia with Landsat 8 images, phenologybased algorithm and Google Earth Engine. Remote Sensing of Environment 185, pp. 142-154.
Guo, Y., Jia, X. and Paull, D., 2017. A domain-transfer support vector machine for multi-temporal remote sensing imagery classification. In: 2017 IEEE International Geoscience and Remote Sensing Symposium (IGARSS), IEEE.

Jin, C., Xiao, X., Dong, J., Qin, Y. and Wang, Z., 2016. Mapping paddy rice distribution using multi-temporal Landsat imagery in the Sanjiang Plain, northeast China. Frontiers of Earth Science 10(1), pp. 49-62.

Kuenzer, C. and Knauer, K., 2013. Remote sensing of rice crop areas. International Journal of Remote Sensing 34(6), pp. 21012139.

Li, P., Jiang, L., Feng, Z., Sheldon, S. and Xiao, X., 2016. Mapping rice cropping systems using Landsat-derived Renormalized Index of Normalized Difference Vegetation Index (RNDVI) in the Poyang Lake Region, China. Frontiers of Earth Science 10(2), pp. 303-314.

Mosleh, M. K., Hassan, Q. K. and Chowdhury, E. H., 2015. Application of remote sensors in mapping rice area and forecasting its production: A review. Sensors 15(1), pp. 769-791.

Nocedal, J. and Wright, S., 2006. Numerical Optimization (Second Edition). Springer Science \& Business Media, LLC.

Qin, Y., Xiao, X., Dong, J., Zhou, Y., Zhu, Z., Zhang, G., Du, G., Jin, C., Kou, W., Wang, J. et al., 2015. Mapping paddy rice planting area in cold temperate climate region through analysis of time series Landsat 8 (OLI), Landsat 7 (ETM+) and MODIS imagery. ISPRS Journal of Photogrammetry and Remote Sensing 105 , pp. 220-233.

Xiao, X., Boles, S., Frolking, S., Li, C., Babu, J. Y., Salas, W. and Moore, B., 2006. Mapping paddy rice agriculture in South and Southeast Asia using multi-temporal MODIS images. Remote Sensing of Environment 100(1), pp. 95-113.

Xiao, X., Boles, S., Liu, J., Zhuang, D., Frolking, S., Li, C., Salas, W. and Moore, B., 2005. Mapping paddy rice agriculture in southern China using multi-temporal MODIS images. Remote Sensing of Environment 95(4), pp. 480-492.

Zhang, G., Xiao, X., Dong, J., Kou, W., Jin, C., Qin, Y., Zhou, Y., Wang, J., Menarguez, M. A. and Biradar, C., 2015. Mapping paddy rice planting areas through time series analysis of MODIS land surface temperature and vegetation index data. ISPRS Journal of Photogrammetry and Remote Sensing 106, pp. 157-171.

Zhou, Y., Xiao, X., Qin, Y., Dong, J., Zhang, G., Kou, W., Jin, C., Wang, J. and Li, X., 2016. Mapping paddy rice planting area in rice-wetland coexistent areas through analysis of Landsat 8 OLI and MODIS images. International Journal of Applied Earth $\mathrm{Ob}$ servation and Geoinformation 46, pp. 1-12. 